\title{
Crush Simulation of Cross-Section Conical Aluminum Alloy 2024-T4 Polyurethane Foam-Filled Section
}

\author{
Azrol Jailani and A. Othman, Member, IACSIT
}

\begin{abstract}
Present paper studies crush simulation of elliptical conical unfilled and foam filled subjected to compressive loading is studied in detail. The finite element analysis package PAM-CRASH 2G version 2005 was compared with existing results from experimental analysis has been done by previous investigation to ensure that the numerical analysis is sufficiently accuracies. The thin-walled extruded aluminum alloy and polymeric foam material offers vast potential for optimally tailoring a design to meet crashworthiness performance requirements. The energy absorption performance of thin-walled extruded 2024-T4 alloy and polymeric foam with variable cross-section in term of vertex angle and wall-thickness are numerically studied. From the results data that achieved from numerical analysis, the load versus deformation curves and analysis dynamic energy absorption versus deformation were plotted and calculated using MathWorks MATLAB Simulink commercial software's package version R2010a. Results showed that the tube's energy absorption capability was affected significantly by varying of velocity-impact and wall-thickness as well as vertex angle of conical cross-section. It is also found that as the filling polymeric foam into thin-walled tube increases the amount of absorbed energy than the empty tubes.
\end{abstract}

Index Terms-Finite element modeling, elliptical conical, axial loading, energy absorption, foam filled.

\section{INTRODUCTION}

Extruded structural aluminum elliptical hollow sections have recently been introduced to the vehicle of aerospace, automotive and marine construction sector [1]-[10]. These structural elements can offer greater structural efficiency than circular hollow sections when subjected to bending or combined loading, or when used as columns with intermediate restraint about the weaker axis, since they possess different major and minor axis flexural properties [3]. Despite recent investigations involving the testing, numerical modeling and development of design rules for elliptical cross-section structure, a number of aspects of their structural response remain unexplored [4]. In particular, the behavior of elliptical profiles in the post ultimate region has not yet been examined. Besides defining the unloading branch of the load-deformation curve [5], an accurate description of this region also allows the possibility of quantifying the ductility of the system. The aim of the present work is to develop a finite element model to predict the full load-deformation

Manuscript received May 15, 2013; revised July 10, 2013

Azrol Jailani is with The Association of Ledang Community Youth, 152 Jalan Puteri 1/6, Bandar Baru Tangkak, 84900 Tangkak, Johor (e-mail: Azrol.jailani@yahoo.com).

A. Othman is with the Mechanical Engineering Department, Polytechnic Port Dickson, KM. 14 Jalan Pantai, 71050, Si Rusa, Port Dickson, Negeri Sembilan, Malaysia (e-mail: akbar.othman@gmail.com). response of elliptical extruded aluminum with and without filling polymeric foam as filler under pure compression. Towards this end, rigid plastic theory has been applied to elliptical profiles based on the method presented. There is comparatively little information available concerning the crushing behavior of core and core-less thin-walled extruded aluminum elliptical shells [6], [7]. But it would appear that no previous investigation has been made in the literature to study the crushing behavior of thin-walled elliptical cones with and without polymeric foam filling. Studies on energy absorption capability as well as the load carrying capacity of thin-walled extruded aluminum elliptical tubes and cones are however still scarce [8]. This was one of the motivating factors behind this paper. The purpose of this study is to investigate and determine the capability and influence of thin-walled extruded aluminum elliptical conical with and without polymeric foam filled as collapsible energy absorber devices.

\section{Finite ELEMENT Modeling}

All models were developed using the nonlinear finite element software PAM CRASH 2G version 2005 [9]. The numerical solutions were also carried out using the explicit finite element code PAM-CRASH 2G. PAM-VIEW was used as the visualization tool postprocessor. The extruded aluminum tubes were modeled using Belytschko-Tsay-4 node-thin shell elements. These elements are three-nodded reduced integration shells with six degrees of freedom per node and are suitable for thick and thin applications. A uniform mesh size of all modeling $3.0 \mathrm{~mm}$ was employed, with maximum element sizes of $5.0 \times 5.0 \mathrm{~mm}$. The foam filler and the compression test plates were modeled with 8 -node solid elements. Finite element meshes of the dynamic axial compression testing of empty and foam-filled tubes are shown in Fig. 1.

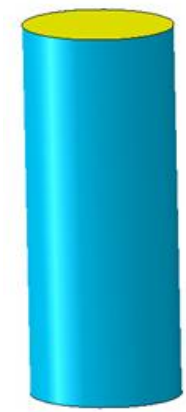

TYPE A

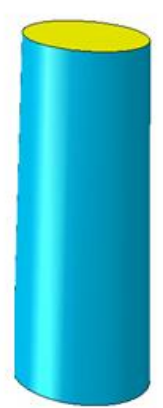

TYPE B

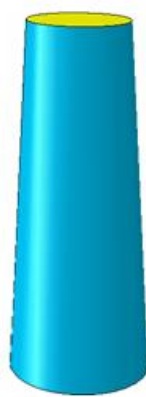

TYPE C
Fig. 1. Finite element model of elliptical conical tubes

The total work done (W) during the axial crushing of the cones are equal to the area under the load/displacement curve 
and is evaluated as:

$$
W=\int P d s
$$

where, $P$ is the force acting on the tube. Therefore the specific energy absorption per unit mass, $E$ is recognized as:

$$
E=\frac{W}{m}
$$

where, $m$ is the crushed mass of the thin-walled tube. It was assumed that aluminum 2024-T4 alloy has only isotropic strain hardening, and for quasi-static loading, the strain rate effects on the yield strength were neglected due to the relatively low overall average load rate used in the tensile tests. For dynamic loading, the effect of strain rate was included in the finite element model using the Cowper-Symonds constitutive equation given by the following relation:

$$
\dot{\varepsilon}_{p}=D^{t}\left(\frac{\sigma_{d}}{\sigma_{s}}-1\right)^{q^{t}} \text { for } \mapsto \sigma_{d} \geq \sigma_{s}
$$

where $\sigma_{d}$ is the dynamic flow stress at a uniaxial plastic strain rate $\varepsilon_{p}, \sigma_{s}$, the associated static flow stress, and the constants $D^{t}$ and $q^{t}$ are tube material parameters. Equation (3) is an overstress power law that was incorporated into the finite element model. The cross-sectional dimensions of the modeled elliptical conical sections were kept constant at $40 \times$ $30 \mathrm{~mm}$, while three variations of wall-thickness of 1.0, 1.5 and $2.0 \mathrm{~mm}$ were considered, providing a range of cross-section slenderness values. The member length was fixed at $150 \mathrm{~mm}$, which was sufficiently short to ensure no global buckling, and all sections were subjected to concentric compression. All models were assigned rigid plastic material properties without strain hardening or residual stresses, to allow direct comparison.

\section{MATERIAL MODEL AND PROPERTIES}

As is shown in Fig. 2, material Type 103 corresponding to the elastic-plastic isotropic thin shell material model was used for the tube material. Material Type 103 uses an enhanced plasticity algorithm that includes transverse shear effects. It exactly satisfies Hill's criterion and precisely updates the element thickness during the plastic deformation. The base and upper plates were modeled using an elastic-plastic solid element model called material Type 1. This material model corresponds to elastic-plastic behavior with isotropic hardening and elastic-plastic behavior can be introduced by specifying the yield stress and tangent modulus or specifying the effective plastic strains and stresses [10].

As is shown in Fig. 3, The material behavior of polyurethane foam was modeled using the crushable foam solid model, namely material Type 2. Material Type 2 corresponds to the solid materials exhibiting coupled volumetric bulk and deviatoric shear plasticity. The coupling between both parts of the material response is established by a pressure dependent von-Mises yield surface. Three contact models were used in the modeling: i) a tied contact between the base plate and the empty tube, ii) a node-to-segment contact between the upper plate and the tube ends and iii) a self-contact (self-impacting contact with edge treatment) in order to prevent the interpenetration between folds in the tube wall during plastic deformation. The self-contact impact algorithm of Type 36 allows all slave segments defined in a given sliding interface. No segment orientation is needed to be specified, since the algorithm automatically detects penetrations and keeps in memory the segment side from which a slave node comes into contact. The self-contact impact algorithm also uses a search algorithm so-called 3D Bucket sort algorithm in which the 3D slave surface is subdivided into a number of buckets and the slave nodes are recalculated in terms of bucket coordinates.

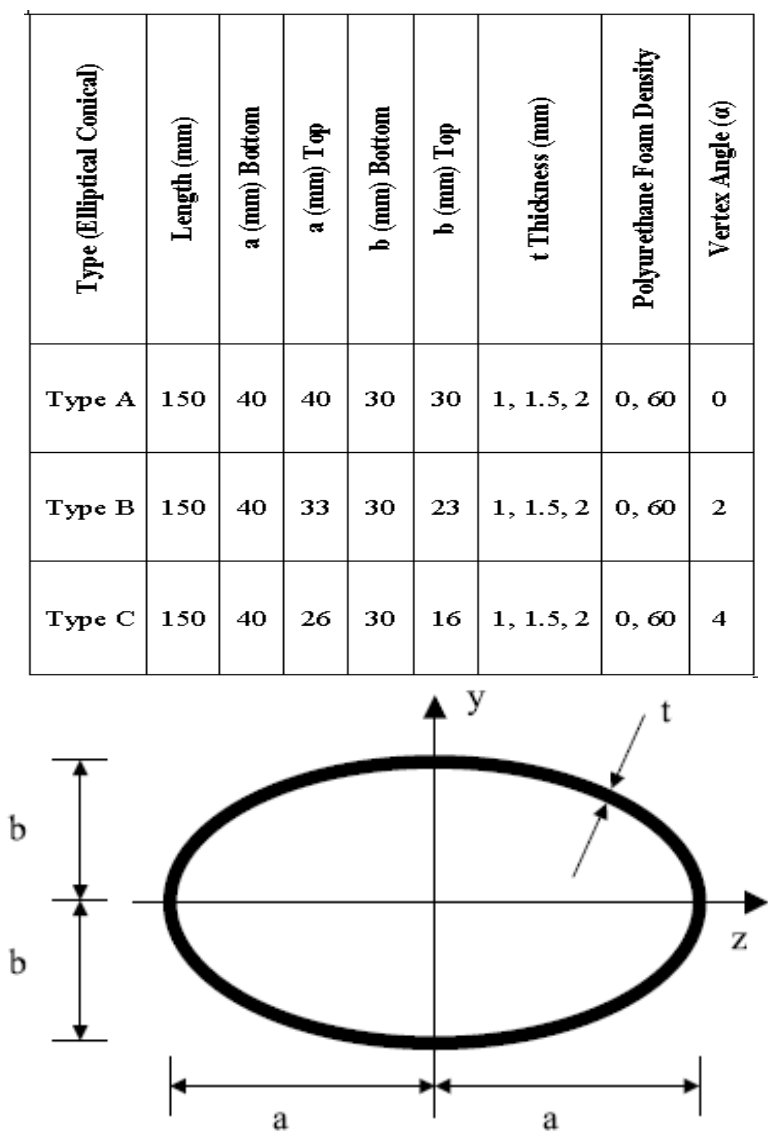

Fig. 2. Dimension of finite element model of elliptical conical tubes.

\section{PARAMETRIC FINITE ELEMENT ANALYSIS}

To carry out real crushing finite element formulation, the value of the velocity and time need to be suitable. The initial velocity ' $v$ ' of the tube are set to be 10,15 and $20 \mathrm{~m} / \mathrm{s}$, hence calculated value for crushing time, ' $t$ ' was found to be $0.02 \mathrm{~s}$. The other important parameter of representing crushing event is boundary condition. In structural analyses, boundary conditions are applied to those regions of the model where there displacements and/or rotations are known. Such regions may be constrained to remain fixed or may have specified nonzero displacements and/or rotations. In this model the fixed-free condition has been used where the top section of the tube is constrained completely and, thus, cannot move in any direction also. The bottom section, however, is fixed in 
the horizontal direction but is free to move in vertical direction. The direction in which motion is possible is called degree of freedom 'dof', hence this model only has a single degree of freedom.

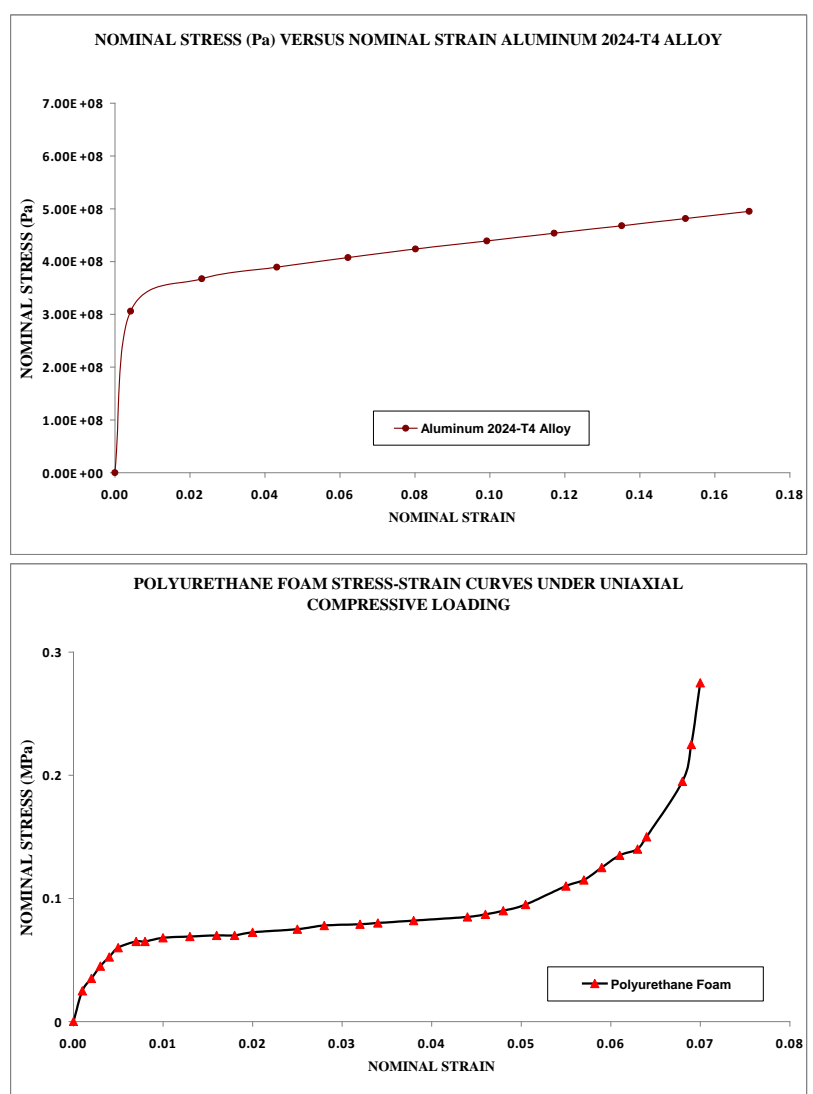

Fig. 3. Nominal stress-strain curve of aluminum 2024-T4 alloy and Nominal stress-strain curve of polyurethane foam.

\section{RESUlt AND DISCUSSION}

The dynamic load displacement curves of empty extruded aluminum elliptical conical tube models constructed with the numbers of elements $3 \times 3 \mathrm{~mm}$, are shown in Fig. 4 to 5 for wall-thickness of 1.0, 1.5 and $2.0 \mathrm{~mm}$ tube, respectively. In these figures, for the purpose of comparison both of deformation crushing element model and dynamic load versus displacement curve of empty extruded aluminum elliptical conical tube is also shown. As seen in Fig. 4 and 5, the dynamic load versus displacement curves of models with deformation crushing of element model on $0,20,40,60,90$, 110,130 and $140 \mathrm{~mm}$ give reasonably good agreements with the different of shape of elliptical conical with 0,2 and 4 degree of vertex angle. The effects of vertex angle on deformation arrangement can be seen in Fig. 4 and 5 which the reduction of dynamic load is after first crash initiator before appeared a folding in surface element model. The lobe of folding were easily be made from level to level deformation of element found corresponding to the points of the first and the last fold formation seen in the Fig. 4 and 5. Finite element models were, however, continued with calculated the crushing process from un-deformed until final progressive collapses. The initial peak load were higher in vertex angle of 0 degree was $7000 \mathrm{~N}$ cause by the frontal impact surface having a large diameter compared to vertex angle of elliptical conical 2 and 4 degree were $3700 \mathrm{~N}$ and
$2500 \mathrm{~N}$, respectively. Whereas having smaller diameter of frontal surface impact will crush progressively without collapse buckling. The agreement between three different of vertex angle of elliptical conical were similarity of average load values. It is quite satisfactory except in the initial and mean load of the load displacement curves, corresponding to the points of the first and the last fold formation.

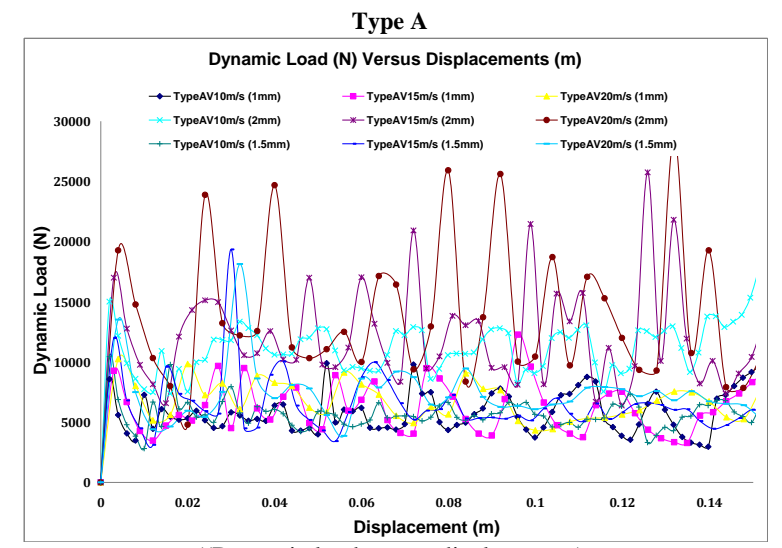

((Dynamic load versus displacement)

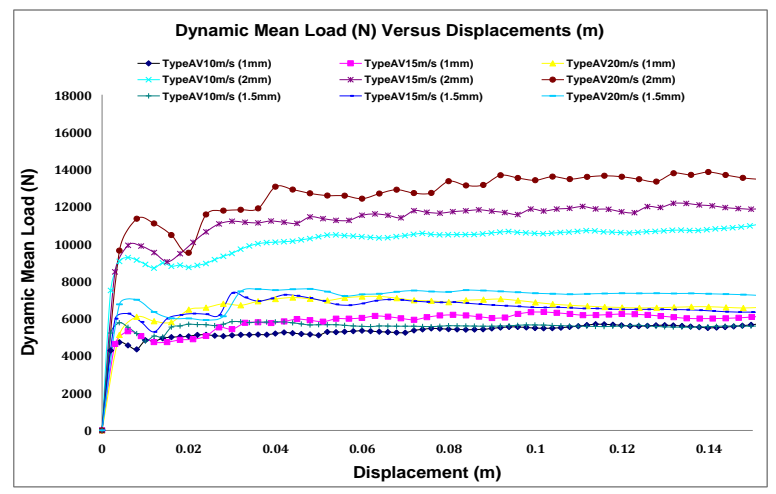

(Dynamic mean load versus displacement)

Type B

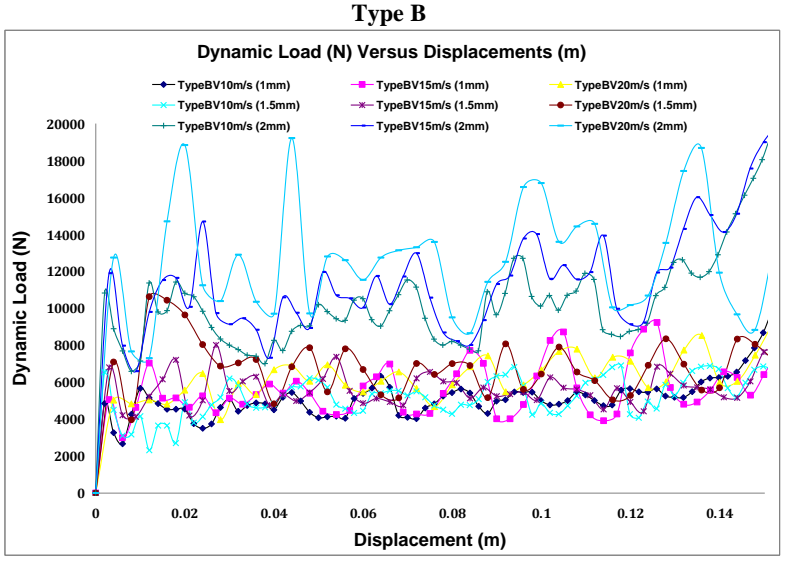

(Dynamic load versus displacement)

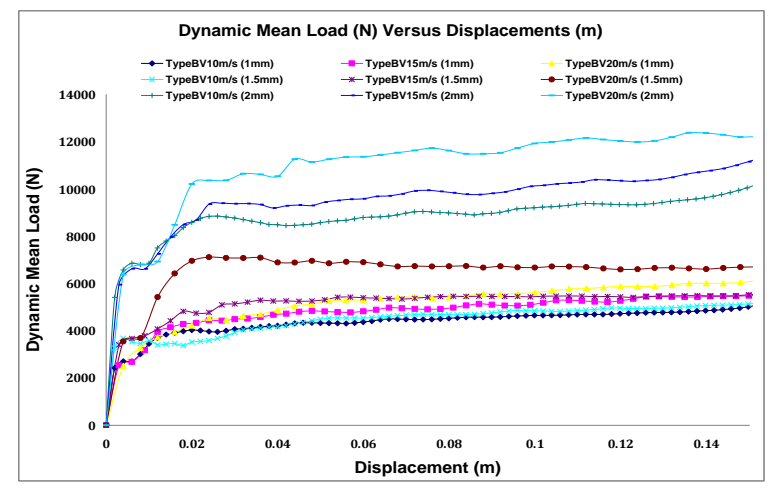

(Dynamic mean load versus displacement) 


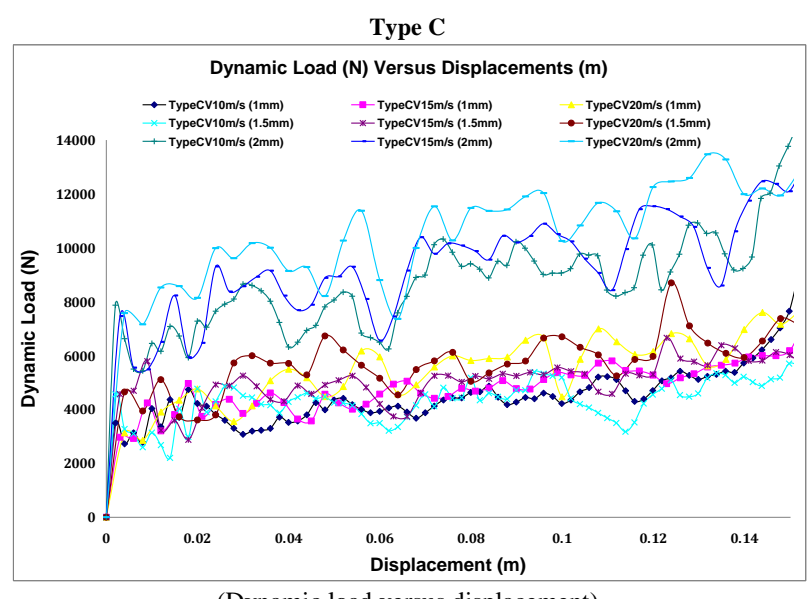

(Dynamic load versus displacement)

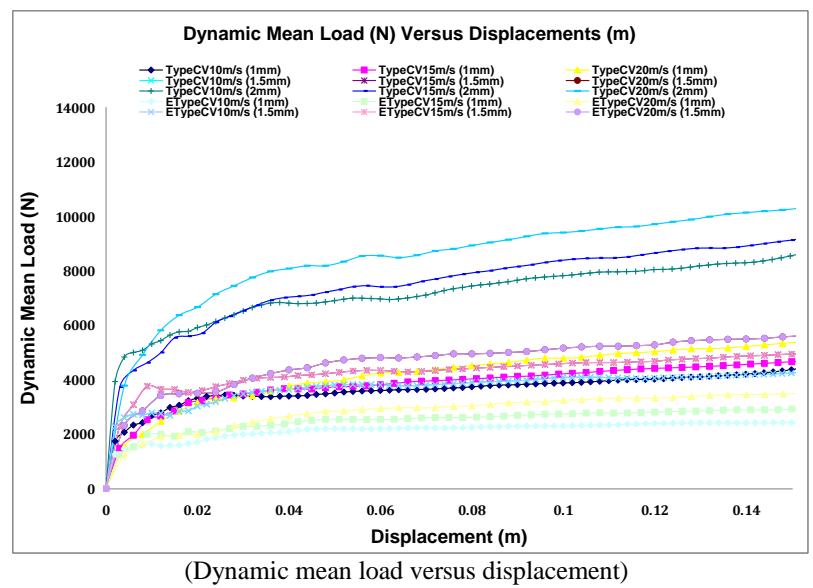

Fig. 4. Dynamic load (N) and dynamic mean load $(\mathrm{N})$ versus displacement (m) elliptical conical foam-filled profile.
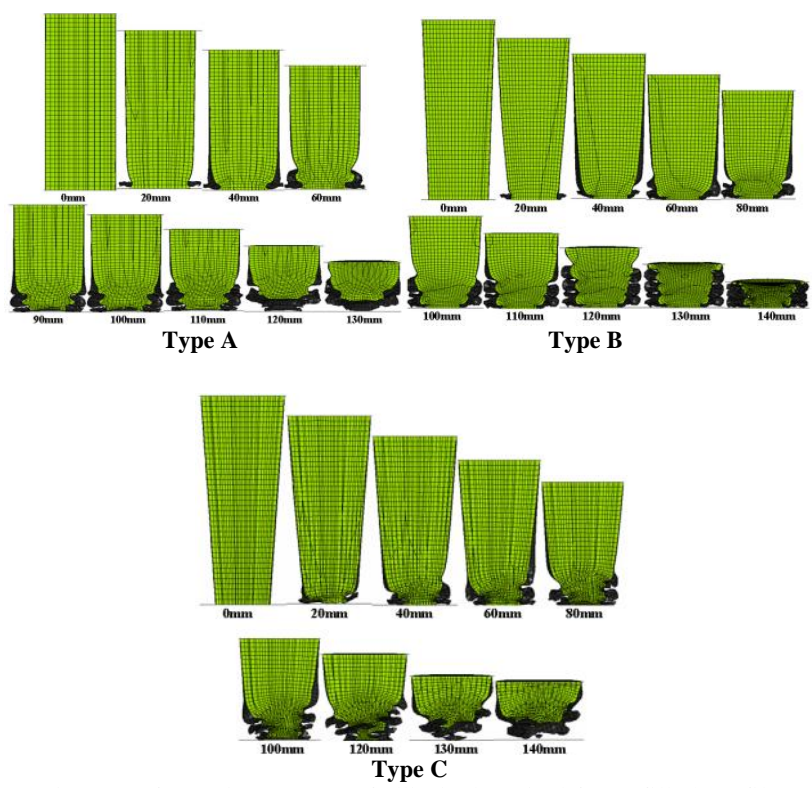

Fig. 5. Deformation pattern of elliptical conical foam-filled profile [Wall-Thickness $=1.0 \mathrm{~mm}]$.

The range element model deformed shapes of empty 130 and $140 \mathrm{~mm}$ elliptical conical corresponding to the deformation ratios of $20 \%, 50 \%$ until $90 \%$ are sequentially shown in Fig. 4 and 5. The density polyurethane foam-filled elliptical conical $60 \mathrm{~kg} / \mathrm{m}^{3}$ was selected in this study and the model resulted in diamond mode of collapse same as the collapse mode of empty elliptical conical. Fig. 4 to 5 show the agreements between the models deformed shapes of tubes at 30 until $150 \mathrm{~mm}$ deformations. The effect of foam filling is to reduce the fold length and hence to increase the number of folds formed a result which was also previously found in foam filled aluminum tubes. The load values of model further show good coincidence with those of numerical analysis as depicted in Fig. 4 to 5.

In contrast all elliptical conical, the foam filling in final deformed tube switched the collapse mode from diamond to concertina mode and again model show very similar deformed shapes load displacement curves in Fig. 4 to 5. The restraining effect of foam filler to the fold formation was explained to be the major cause of the reduced fold length in filled tubes. The entrance of the elliptical conical column wall into foam filler results in shorter fold lengths and increases the number of fold formed. Santosa et al stated that the encroachment of the column wall into the aluminum foam filler allows an additional compression in the foam and retards the sectional collapse of the column [9], [10]. A similar filler elliptical conical encroachment effect is also seen on the surface images of partially crushed foam-filled tubes fillers and tubes in Fig. 4 to 5. Polyurethane foam filled aluminum tubes was proposed to be due to the thickening effect of foam filling, which drives the deformation shift from diamond to concertina. It was also shown by Hanssen et al. that after a critical aluminum foam density the deformation mode in filled aluminum tubes shifted from diamond to concertina mode. A similar mode-shift was also found in polyurethane foam-filled thin-walled aluminum tubes with the increasing of foam density.

\section{CONCLUSION}

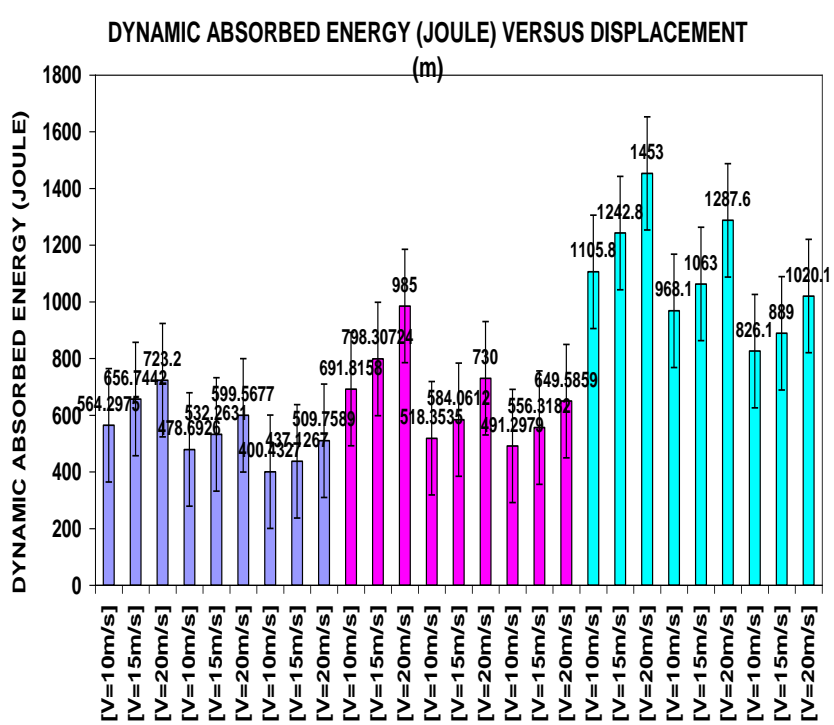

Fig. 6. Dynamic absorbed energy (Joule) Type of elliptical conical Type of A, $\mathrm{B}$ and $\mathrm{C}$ foam-filled profile [Wall-Thickness $=1.0,1.5$ and $2.0 \mathrm{~mm}$ ] (Velocity $=10$ and $20 \mathrm{~m} / \mathrm{s}$ ).

As is shown in Fig. 6, the dynamic crushing behavior of extruded polyurethane foam-filled aluminum 2024-T4 alloy extrusion elliptical conical tubes was investigated numerically. The numerical solutions were carried out using the explicit finite element code PAM-CRASH 2G version 2005. The kinetic and internal energy histories were investigated at different mass density scaling and deformation velocities and based on efficiency and time inexpensive modeling a mass density scaling with a factor of 
1000 and a loading velocity of 10,15 and $20 \mathrm{~m} / \mathrm{s}$ were chosen for the modeling. In general, satisfactory agreements were found between the results of finite element modeling and experimental dynamic crushing tests of foam-filled thin-walled aluminum tubes.

The model has also highlighted several effects of foam filling in thin-walled elliptical conical aluminum 2024-T4 alloy extrusion tubes. The number of folds formed in foam-filled tubes, both in diamond and concertina mode of deformation, increased with foam filling and also with increasing foam filler density. The energy absorptions in foam-filled tubes were shown to increase with increasing filler density and higher than the sum of the energy absorptions of empty tube (alone) and filler (alone). The type of $\mathrm{C}$ for empty and foam-filled profile exhibited the highest performance of absorbed energy. Secondly introducing the elliptical angle shape of conical profile will reduce the initial peak load and thoroughly the damage of thin-walled structure will failure with progressively. Lastly introducing the foam filler into empty thin-walled section can improve the absorbed energy either any cross-section or the type of filler material.

\section{REFERENCES}

[1] A. Reyes and M. Langseth et al., "Crashworthiness of aluminium extrusions subjected to oblique loading: experiments and numerical analyses," Int J. Mech Sci, vol. 44, no. 19, pp. 65-84, 2002.

[2] J. M. Alexander, "An approximate analysis of collapse of thin-walled cylindrical shells under axial loading," Q. J. Mech Appl Math, vol. 13 pp.1-9, 1960.

[3] A. A. A. Alghamdi, "Collapsible impact energy absorbers: an overview,” Thin Wall Struct, vol. 39, pp. 189-213, 2001.

[4] B. H. Lampinen and R. A. Jeryan, "Effectiveness of polyurethane foam in energy absorbing structures," SAE Paper 820494, 1982.

[5] S. R. Reid, T. Y. Reddy and M. D. Gray, "Static and dynamic axial crushing of foam filled sheet metal tubes," Int J. Mech Sci, vol. 23, pp. 295-322, 1986.

[6] S. R. Guillow, G. Lu, and R. H. Grezbieta, "Quasi-static compression of thin-walled circular aluminum tubes," Int J, Mech Sci, vol. 43, no. 210, pp. 3-23, 2001.

[7] T. Y. Reddy and R. J. Wall, "Axial compression of foam filled thin-walled circular tubes," Int J. Impact Eng., vol. 7, no. 1, pp. 51-66, 1988.

[8] M. Seitzberger, F. G. Rammerstorfer, R. Gradinger, H. P. Degischer, M. Blaimschein, and C. Walch, "Experimental studies on the quasi-static axial crushing of steel columns filled with aluminum foam," Int $J$. Solids Struct, vol. 37, no. 41, pp. 25-47, 2000.

[9] S. Santosa and T. Wierzbicki, "Crash behavior of box columns filled with aluminum honeycomb or foam," Comput Stuct, vol. 68, no. 3, pp. 43-67, 1998.

[10] S. Santosa, T. Wierzbicki, A. G. Hanssen, and M. Langseth, "Experimental and numerical studies of foam-filled sections," Int J. Impact Eng., vol. 24, no. 50, pp. 9-34, 2000.

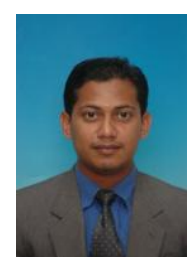

Azrol Jailani is a member of IAENG (121660) and he was born in Johor, Malaysia on $5^{\text {th }}$ February. He got a first degree in Mechanical Engineering with honors from Universiti Tun Hussein Onn Malaysia in 2007. He is currently pursuing studies in the same field in postgraduate program at Universiti Tun Hussein Onn Malaysia.

$\mathrm{He}$ is currrently an independent researcher at the association of Ledang Community Youth, Johor, Malaysia. Previously he had served in Ledang Community College and Port Dickson Polytechnics, Malaysia as a lecturer. He is interested in the field of materials science and many research he has published into international journal.

Engr. Jailani is member of Institute of Engineers, Malaysia (28618), Board of Engineers Malaysia (53480A), International Association Computer Science \& Information Technology (80344528) and Institute of Materials Malaysia (4109). Paper "Progressive Damage of Double-Cell Square Aluminium 6061-T5 Alloy Polystyrene Foam-Filled Section" gets the best paper awards at 2nd. International Conference on Arts, Social Sciences \& Technology (iCAST2012), 3rd. - 5th. March 2012, Penang, Malaysia.

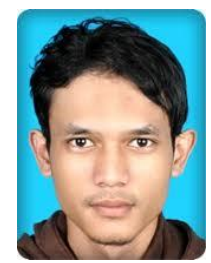

Akbar Othman is a lecturer of Mechanical Engineering at Port Dickson Polythecnic. He received his first degree in Mechanical Engineering from Universiti Tun Hussien Onn Malaysia in 2006, then he continued studies at Universiti Putra Malaysia (UPM) in Structure and Design Engineering, leading to Master degree. $\mathrm{He}$ is working on simulating crushing performance of hybrid laminated material Tapered Tubes under oblique loading conditions. He was awarded with a M. Sc. degree in 2009. After that, his has been pursuing his Ph.D degree in Quasi-Static and Energy Absorption Performance of Foam-Filled Composite Tubes under Off-axis Loading at Universiti Kebangsaan Malaysia, (UKM) in progress. This author became a member (M) of IAENG, International Association of Computer Science and Information Technology (IACSIT) and board of Engineer Malaysia (BEM). 